\title{
Identification and Analysis of Potential Autophagy-Related Biomarkers in Endometriosis by WGCNA
}

\author{
Jing Wang ${ }^{1}$, Shanshan Cong ${ }^{1}$, Han Wu ${ }^{1}$, Yanan $\mathrm{He}^{1}$, Xiaoli Liu ${ }^{2}$, Liyuan Sun ${ }^{1}$, Xibo Zhao ${ }^{1}$ and \\ Guangmei Zhang ${ }^{1 *}$ \\ ${ }^{1}$ Department of Gynecology, The First Affiliated Hospital of Harbin Medical University, Harbin, China, ${ }^{2}$ Department of Gynecology, \\ The Red Cross Center Hospital of Harbin, Harbin, China
}

OPEN ACCESS

Edited by:

Xin Zhang,

Jiangmen Central Hospital, China

Reviewed by:

Dong Ren,

The First Affiliated Hospital of Sun Yat-sen University, China

Hui Liu,

Changzhou University, China

${ }^{*}$ Correspondence:

Guangmei Zhang

guangmei_zhang@126.com

Specialty section:

This article was submitted to

Molecular Diagnostics and

Therapeutics,

a section of the journal

Frontiers in Molecular Biosciences

Received: 17 July 2021

Accepted: 11 October 2021

Published: 01 November 2021

Citation:

Wang J, Cong S, Wu H, He Y, Liu X,

Sun L, Zhao $X$ and Zhang $G$ (2021)

Identification and Analysis of Potential

Autophagy-Related Biomarkers in

Endometriosis by WGCNA.

Front. Mol. Biosci. 8:743012.

doi: 10.3389/fmolb.2021.743012
Background: Endometriosis is a serious gynecological disorder characterized by debilitating pain, infertility and the establishment of innervated endometriosis lesions outside the uterus. Early detection and accurate diagnosis are pivotal in endometriosis. The work screened autophagy-related genes (ATGs) as potential biomarkers to reveal new molecular subgroups for the early diagnosis of endometriosis.

Materials and Methods: The gene lists of ATGs from five databases were integrated. Then, weighted gene co-expression network analysis (WGCNA) was used to map the genes to the gene profile of endometriosis samples in GSE51981 to obtain functional modules. GO and KEGG analyses were performed on the ATGs from the key modules. Differentially expressed ATGs were identified by the limma R package and further validated in the external datasets of GSE7305 and GSE135485. The DESeq2 R package was utilized to establish multifactorial network. Subsequently, one-way analysis of variance (ANOVA) was performed to identify new molecular subgroups. Real-time quantitative polymerase chain reaction (RT-qPCR) and Western blotting were used to confirm the differential expression of hub ATGs, and the receiver operating characteristic (ROC) curve analysis and Spearman correlation analysis were applied to assess the diagnostic value of hub ATGs in 40 clinical samples and human primary endometrial stromal cells (ESCs).

Results: We screened 4 key modules and 12 hub ATGs and found the key genes to be strongly correlated with endometriosis. The pathways of ATGs were mainly enriched in autophagy, apoptosis, ubiquitin-protein ligase binding, and MAPK signaling pathway. The expression levels of EZH2 (Enhancer of Zeste homolog 2) and RND3 (also known as RhoE) had statistically significant changes with higher values in the endometriosis group compared with the controls, both in the tissue samples and primary ESCs. Besides, they also showed higher specificity and sensitivity by the receiver operating characteristic analysis and Spearman correlation analysis for the diagnosis of endometriosis. The TFmRNA-miRNA-IncRNA multifactorial network was successfully constructed. Four new molecular subgroups were identified, and we preliminarily showed the ability of IQCG to independently differentiate subgroups.

Conclusion: EZH2 and RND3 could be candidate biomarkers for endometriosis, which would contribute to the early diagnosis and intervention in endometriosis. 
Keywords: endometriosis, autophagy-related genes (ATGs), weighted gene co-expression network analysis (WGCNA), EZH2, RND3

\section{INTRODUCTION}

Endometriosis is a chronic, inflammatory, oestrogen-dependent disease, in which endometrial stromal cells and glands are implanted outside the uterine cavity (Bulun et al., 2019). It affects approximately $10 \%$ of the women of reproductive-age and leads to a marked reduction in the patients' quality of life (QoL) (Vallvé-Juanico et al., 2019). So far, no consistent theory regarding the etiological factors of endometriosis has been established. In addition, it is hard to be diagnosed at early stages, and the available clinical methods are limited.

Recent studies have indicated that autophagy plays an integral role in the physiological and pathophysiological mechanisms associated with the endometria, including the menstrual cycle, decidualization and vascular remodeling of normal pregnancy, as well as endometrium-related diseases, such as endometriosis, infertility or subfertility, and endometrial carcinoma (Yang et al., 2017; Yang et al., 2019b). In consistence with the changes in autophagy levels between ectopic and eutopic endometria in endometriosis, an aberrant expression of autophagy-related genes has also been observed (Luo et al., 2018; Mei et al., 2018). Many factors have been shown to contribute to abnormal autophagy in endometrial tissues, including estrogen, certain drugs, oxidative stress, and hypoxia (Rižner, 2009; Zhao et al., 2018). Besides, autophagy has been connected with the recurrence and development of endometriosis (Ma et al., 2019; He et al., 2020). Large-scale screening and bioinformatics tools revealed the abnormal expression of ATGs to be closely related to the initiation, development, early diagnosis and poor prognosis of tumors, such as gastric carcinoma, prostate cancer, and lung cancer (Yepes et al., 2016; Huang et al., 2017). However, the connection between hub ATGs and endometriosis still needs to be exploited.

Weighted gene co-expression network analysis (WGCNA) represents one of the most important and widely used methods of systems bioinformatic (Langfelder and Horvath, 2008), which is used to describe the correlation patterns among genes via microarray specimen (Langfelder and Horvath, 2012). It can be executed through an R package to group the genes into modules according to the gene co-expression similarities across the samples. The WGCNA method can not only retrieve a cluster of genes with similar biological functions, but it may connect modules to external clinical features. As a result, relevant functional networks can be used to identify specific biomarkers and new molecules (Liu et al., 2017; Pascut et al., 2020).

In this work, we present an innovative investigation of the association between ATGs and the endometriosis clinical traits. EZH2 and RND3 were proven to be candidate diagnostic biomarkers and validated by external datasets and experiments. Four new molecular subgroups were identified, and a multifactorial network was constructed. The insights
TABLE 1 | Sample information of endometriosis in database.

\begin{tabular}{lcccc}
\hline & Sample & Control & Endometriosis & All \\
\hline mRNA & GSE51981 & 71 & 77 & 148 \\
mRNA & GSE135485 & 4 & 54 & 58 \\
mRNA & GSE7305 & 10 & 10 & 20 \\
miRNA & GSE105765 & 8 & 8 & 16 \\
IncRNA & GSE105764 & 8 & 8 & 16
\end{tabular}

from this work lay a theoretical foundation for the early diagnosis and prompt treatment of endometriosis.

\section{MATERIALS AND METHODS}

\section{Data Preparation}

We used the Gene Expression Omnibus (GEO, https://ncbi.nlm. mih.gov/geo/), which is a public functional genomics database with various types of gene profiles in various diseases. We retrieved multiple GEO series, including mRNA (GSE51981, GSE135485, GSE7305), miRNA (GSE105765), and lncRNA (GSE105764) datasets. Details of the retrieved data are listed in Table 1. Then, we integrated the ATGs derived from HADb (http://www.autophagy.lu/index.html),

AUTOPHAGY DATABASE (http://www.tanpaku.org/autophagy/index.html), HAMdb (http://hamdb.scbdd.com/), GSEA (https://www.gseamsigdb.org/gsea/index.jsp) and NCBI (https://www.ncbi.nlm. nih.gov/). A total of 2,209 genes were extracted, among which 1928 autophagy-related genes were expressed in the GSE51981 dataset (See Supplemental Table S1).

\section{Weighted Gene Co-Expression Network Analysis}

We applied the WGCNA method to construct the gene coexpression network and identify the functional modules (Langfelder and Horvath, 2008). Hierarchical clustering analysis was performed using the $\mathrm{R}$ software package gplots and the sample outliers were identified. The WGCNA algorithm was used to calculate the ATGs correlation among the samples. Then the appropriate soft threshold power was performed, and the standard scale free network was established. The models or networks that were obtained using WGCNA were relevant to the external sample features. Next, the correlation functional networks were used to identify tissuespecific markers. The modules were identified using a dynamic tree-cut strategy by clustering the genes hierarchically. Next, WGCNA was used to construct the gene co-expression modules and extract the gene information in each module. A total of 1928 autophagy-related genes (ATGs) were mapped to the expression profile of the GSE51981 samples, and key functional modules with highly correlated genes were identified. 


\section{Enrichment Analysis of Key Modules and Identification of Hub ATGs}

According to the obtained gene significance scores, the numbers of genes in key modules were arranged in sequence from high to low. Next, functional enrichment analysis was performed to analyze, identify, and interpret diverse biological functions based on the Kyoto Encyclopedia of Genes and Genomes (KEGG) pathway analysis and Gene Ontology (GO) annotation analysis in the functionally related modules. The data were visualized and analyzed using the ClusterProfiler and ggplot2 $\mathrm{R}$ packages (pvalueCutoff $=0.05$ ).

Hub genes represent the top-ranked genes in the intermodular connectivity, which are centrally located in their respective module and can reflect its characteristics. Compared with the other genes in the network, the hub genes of key modules have a greater biological significance. Using the limma R package, differential expression analyses were performed between the endometriosis group and control group on the GSE51981 dataset $(p<0.05)$ (Ritchie et al., 2015). WGCNA was used to identify gene-gene interactions in each notable module, and the hub ATGs were then predicted using CytoHubba (Degree method, Rank <1) (Chin et al., 2014). The above-mentioned data were merged, and the overlaps were calculated. In order to further confirm the significance and accuracy of the screening genes, the expression of the resulting hub ATGs were validated using two external datasets: GSE7305 (ten control samples, ten endometriosis samples) and GSE135485 (four control samples, fifty-four endometriosis samples).

\section{New Molecular Subgroups and Multifactorial Network}

Based on the expression of hub ATGs in 77 samples from GSE51981, we performed a clustering analysis of the dataset using the ConsensusClusterPlus $\mathrm{R}$ package to recognize new molecular subgroups (Wilkerson and Hayes, 2010). As a result, we obtained the item-consensus results and cluster consensus. One-way analysis of variance (ANOVA) was used to determine whether the expression of the hub ATGs significantly varied among the molecular subgroups (ANOVA, $p<0.05)$.

We retrieved the miRNA and mRNA interactions from the miRNA 3.0 database (http://mirwalk.umm.uni-heidelberg. $\mathrm{de} /$ ), under the condition that the interaction relationships were predicted from all four databases (miRNA, TargetSan, miRDB, miRTarBase). We retrieved the interaction relationship between miRNA and lncRNA from the StarBase v3.0 database (http://starbase.sysu.edu.cn) and the relationship between the TF and mRNA from the TRRUST v2 database (http://www.grnpedia.org/trrust/). Finally, we used the DESeq2 R package (Love et al., 2014) to analyze the differential expression of miRNA and lncRNA.
TABLE 2 | Clinical Characteristic of tissue samples.

\begin{tabular}{lcc}
\hline Characteristic & Endometriosis $(\boldsymbol{n}=\mathbf{2 0})$ & Control $(\boldsymbol{n}=\mathbf{2 0})$ \\
\hline $\begin{array}{l}\text { Age } \\
\text { Main Diagnose }\end{array}$ & $36.8 \pm 4.6$ & $33.5 \pm 3.5$ \\
& Ovarian Endometriosis & Idiopathic Infertility
\end{tabular}

phases of the menstrual cycle

\begin{tabular}{lcc}
\hline Proliferation phase & 14 & 15 \\
Secretory phase & 6 & 5
\end{tabular}

Secretory phase

Pretherapy clinical staging

Stage I-II

Stage III-IV

12 NA

Distribution of Endometriosis

Ovarian Endometriosis

Dysmenorrhea

Peritoneal Lesion

13

4

Pelvic adhesion

President

Absent

13

7

9

DIE status

With DIE lesions

Without DIE lesions

5

15

NA

CA125 (mean \pm SD)

Stage I-II

Stage III-IV

$85.6 \pm 15.3$

$108.4 \pm 16.7$

NA

NA: not applicable.

DIE: deep infiltrating endometriosis.

\section{Collection of Tissue Samples and Isolation of Human Primary ESCs}

Twenty female patients aged 29-46 years who underwent surgery for endometriosis (endometriosis group) and twenty female patients aged 26-43 years who underwent surgery for idiopathic infertility (control group) in the First Affiliated Hospital of Harbin Medical University (Harbin, PR China) were enrolled in the study. The ectopic endometrium tissues were obtained from the endometriosis group and the normal endometrial tissues were obtained from the control group. Detailed clinical characteristics of all samples were summarized in Table 2. All the patients provided an informed consent form before recruitment and the research protocol was approved by the Ethics Commission of Harbin Medical University (202105).

Primary normal endometrial stromal cells (NESCs) and ectopic endometrial stromal cells (EESCs) were isolated from fresh tissues according to the previously published methods (Gargett et al., 2009). Tissues were transported to the laboratory in an ice-cold medium (DMEM/F-12 1:1) within $2 \mathrm{~h}$, washed twice with sterile PBS to remove the blood and then minced into small pieces and digested in type IV collagenase (Life Technologies) at $37^{\circ} \mathrm{C}$ for $60-90 \mathrm{~min}$. The cell 
TABLE 3 | Primer sequences for RT-qPCR.

Forward

GGACCACAGTGTTACCAGCAT

TCCCTTCCAGATGTGCTGAG

GCGTGGTGGTCCATGTATGT

GCTCCATGTCTTCGCCAAG

CAGAGGCTGTTGACAGAGCA

GTGACCGATGAACCTCCCAAA
Reverse

GTGGGGTCTITATCCGCTCAG ACGGGCATGATGTAGTTCAGA TGAGGTGGATCGGCGTGTA AAAACTGGCCGTGTAATTCTCA TCACTAGGAGGGAGAGTTCTGA CCAGGTCTGCACACTTGACAG suspension was obtained after filtering through a 70- $\mu \mathrm{M}$ sieve to remove debris and glandular epithelial cells. The ESCs suspension was collected and centrifuged at $800 \mathrm{rpm}$ for $5 \mathrm{~min}$ at room temperature. The isolated cells were maintained in the medium (DMEM/F-12 1:1) containing $10 \%$ fetal bovine serum and $1 \%$ antibiotic penicillinstreptomycin (Gibco) at $37^{\circ} \mathrm{C}$ and $5 \% \mathrm{CO} 2$. The culture medium was changed every 3 days. The purity of ESCs was detected by separate immunofluorescence for the epithelial marker Cytokeratin 7 (Abcam) and stromal marker Vimentin (Abcam). Only the study that measured Vimentin positive cells at more than $95 \%$ were included.

\section{Real-Time Quantitative Polymerase Chain Reaction}

Total RNA was extracted from the patients' specimens and human primary ESCs using Trizol reagent (Invitrogen, United States), and it was reverse transcribed to cDNA using a reverse transcription kit (TaKaRa, Japan) following the provided instructions. Quantitative PCR was performed using SybGreen by the real-time PCR detection system (Yang et al., 2019a). A total volume of $20 \mathrm{ul}$ was subjected to the PCR process: $95^{\circ} \mathrm{C}$ for $30 \mathrm{sec}$, 40 cycles of $95^{\circ} \mathrm{C}$ for $5 \mathrm{sec}$, and $60^{\circ} \mathrm{C}$ for $30 \mathrm{sec}$. Then, relative mRNA levels were calculated using the comparative CT method $\left(2^{-\triangle} \mathrm{Ct}\right.$ ) (Zhou et al., 2018). Primer sequences used in the study are described in Table 3.

\section{Western Blotting Analysis}

Total protein was extracted from both the tissues and cells following the instructions of the RIPA lysis buffer (Sigma, United States). Next, total protein concentration was measured using the BCA assay (Beyotime, China). Total proteins (30 ug) were separated on a $4-12 \%$ polyacrylamide gel by SDS-PAGE and electrophoretically transferred onto PVDF membranes, subsequently sealed with $5 \%$ BSA. The membranes were incubated with diluted primary antibodies to EZH2 (1:2,000 dilution, Abcam), RND3 (1:1,000 dilution, Abcam), IQCG (1:1,000 dilution, Abcam) overnight at $4{ }^{\circ} \mathrm{C}$. The membrane was then further incubated with HRP-labelled secondary antibodies (Jackson, United States) at room temperature for $1 \mathrm{~h}$. Finally, protein bands were analyzed and quantified using the ImageJ software.

\section{Statistical Analysis}

All statistical analyses were established using GraphPad Prism 8.0 and SPSS v20.0. The statistical significance of pairwise differences between the groups was indicated by an unpaired $t$-test, and a $p \leq$ 0.05 was considered to be statistically significant. The receiver operating characteristic (ROC) curve analysis and Spearman correlation analysis were used to evaluate the diagnostic value of the hub ATGs and the correlation of the hub ATGs with cancer antigen 125 (CA125).

\section{RESULTS}

\section{Selection of Key Modules}

In our study, seven modules (indicated by the colors of Blue, Brown, Yellow, Red, Green, Turquoise and Grey) were identified according to the average linkage hierarchical clustering (Figure 1A, see Supplemental Table S2 for the number of genes in each module). The analysis of the relationship between the gene co-expression modules and clinical traits resulted in several findings. The genes in the Brown module were positively correlated with the clinical traits of the control group and negatively correlated with those of the endometriosis group. However, the Red, Green, Turquoise, and Grey modules were negatively correlated with the clinical features of the control group and positively related to those of the endometriosis group (Figure 1B). At last, four key modules (the Brown, Turquoise, Green, and Red modules) were identified. The heat map based on the co-expressed genes showed that ATGs in the same model tended to have a high biological relevance (Figure 1C).

\section{GO and KEGG Enrichment Analysis}

We visualized the top ten pathways resulting from the GO and KEGG pathway analysis. The analysis resulted in 254 ATGs in the Brown model, as shown in Figures 2A-D. They mainly focused on the signaling pathways associated with the components of autophagy, apoptosis, ubiquitin-protein ligase binding, and MAPK signaling pathway. In addition, the analysis resulted in 589 ATGs in the Turquoise module, as shown in Figures 3A-D, which focused on autophagy and protein serine/threonine kinase activity pathway. In the Green model, 35 ATGs resulted from the analysis, as shown in Supplemental Figures 1A-C, which were mainly related to the inflammatory mediator regulation of TRP channels. Finally, in the Red module, the analysis resulted in 28 ATGs, as shown in Supplemental Figures 1D-F, which were mainly enriched in cell cycle. 

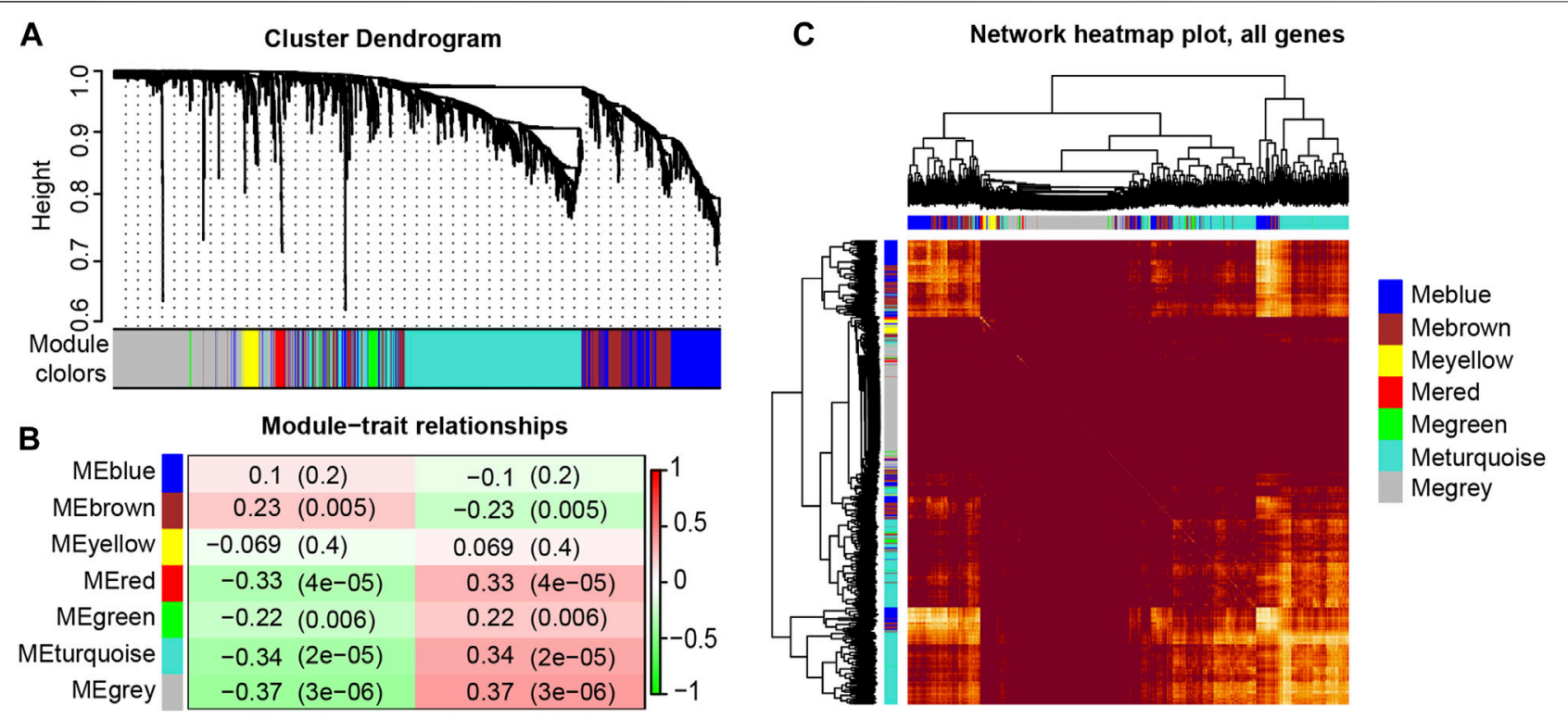

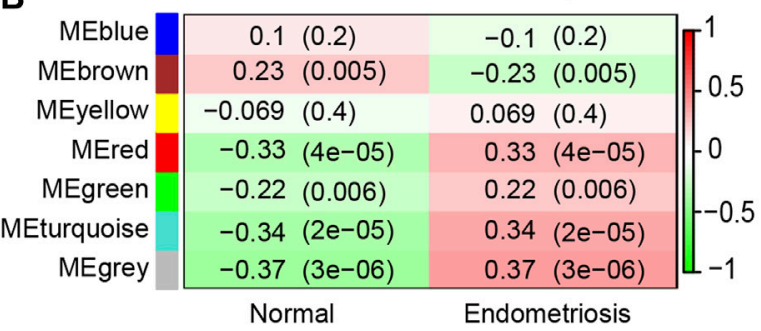

FIGURE 1 | Identification and characterization of endometriosis-associated autophagy-related genes (ATGs) key modules. Hierarchical cluster tree showing coexpression modules based on WGCNA (A). Correlation between seven key modules and clinical traits (B). Co-expression heat map and correlations for ATGs in models (C).

\section{Identification and Validation of 12 Hub ATGs in External Datasets}

We screened the ATGs of key modules and retained co-expressed gene pairs. Then, hub genes obtained by the cytoHubba were overlapped with the differentially expressed ATGs in GSE51981 (Supplemental Table S3). As a result, we obtained 12 hub ATGs (TSIX, FKBP8, EZH2, SPATA18, IQCG, PLK2, RCAN1, RND3, PPP2R5E, EIF4G3, EPHA5, AURKA), the typical box plot is shown in Figure 4A. Notably, the expression level of each hub ATG was significantly higher in the endometriosis group compared with the control group $\left({ }^{* *} p<0.001\right)$. Then, we validated the 12 hub ATGs in the two external datasets of GSE7305 and GSE135485. The comparison of the expression levels of the hub ATGs between the endometriosis group and control group is shown in Supplemental Figures 2A,B, normalized by $\log 2(n+1)$. These validation results were also roughly consistent with the results obtained from GSE51981.

\section{New Molecular Subgroups in Endometriosis}

We mapped 1928 ATGs to the gene expression profile of endometriosis GSE51981, which was performed by consensus clustering analysis. Endometriosis samples were appropriately classified into four subgroups, as shown in Figure 4B (At $\mathrm{K}=4$, cluster heat map). Our results showed 21, 18, 14, and 24 samples in cluster 1 , cluster 2 , cluster 3 , and cluster 4 , respectively. We also analyzed the expression of the 12 hub ATGs in the different clusters (Figure 4D). The distribution of the four new molecular subgroups in different periods of the uterus endometrium is illustrated by a Circos plot in Figure 4C, in this figure, four subgroups are shown in the inner circle, corresponding to different periods of endometrium samples in the outer circle. Statistical comparisons of the 12 hub ATGs using one-way ANOVA resulted in four significantly different ATGs $(p<$ $0.05)$. Then, we further investigated the expression levels of two representative genes (FKBP8, IQCG) in different clusters (Supplemental Table S4). The results showed that both FKBP8 and IQCG were obviously differentially expressed among the four subgroups (Figure 5A). The above-mentioned results suggested that FKBP8 and IQCG could potentially serve as independent factors to discriminate between different subgroups.

\section{A Multifactorial Network Construction}

A total of 27 miRNA-hub ATGs were acquired from the miRwalk 3.0 database according to the interaction relationship in $3^{\prime}$ UTR, 5 ' UTR and CDS regions (Supplemental Table S5). Besides, 26 miRNA-lncRNA interaction relationships were obtained based on the StarBase v3.0 database (Supplemental Table S6), and 28 TFmRNA interaction relationships were retrieved from the TRRUST v2 database (Supplemental Table S7). The analysis of variance on GSE105765 and GSE105764 using the R package DESeq2 resulted in six IncRNA and four miRNA that were overlapped between the above-mentioned analysis results and network relationships. Thus, we successfully constructed the TF-mRNA-miRNA-lncRNA multifactorial network, as shown in Figure 5B.

\section{ROC Analysis and Spearman Correlation Analysis}

The ROC analysis was employed to evaluate the specificity and sensitivity of the 12 hub ATGs for the diagnosis of endometriosis. The accuracy of the score was assessed by calculating the ROC curve 
A

\section{है}

B

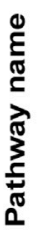

C

$\stackrel{0}{\varepsilon}$
$\frac{\pi}{5}$
$\frac{\pi}{\pi}$
$\frac{1}{5}$
$\frac{5}{0}$
0

D

हั
Top10 of GO:BP enrichment

positive regulation of cellular catabolic process

positive regulation of I-kappaB kinase/NF-kappaB signaling

positive regulation of catabolic process

regulation of macroautophagy

regulation of I-kappaB kinase/NF-kappaB signaling

I-kappaB kinase/NF-kappaB signaling

regulation of autophagy

macroautophagy

process utilizing autophagic mechanism

autophagy

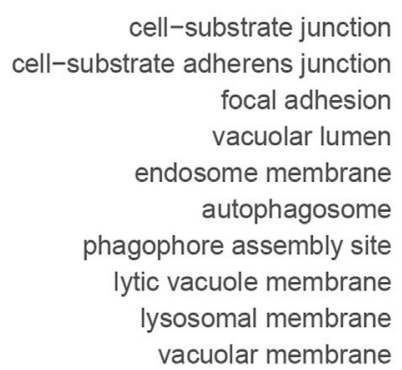

vacuolar membrane

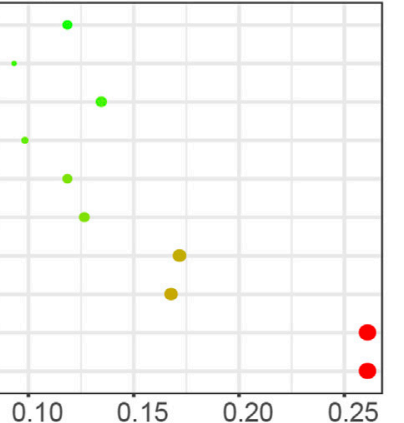

Gene number

- 30

$\circ 40$

○ 50

○ 60

$-\log 10$ (pvalue)

\section{Top10 of GO:CC enrichment}

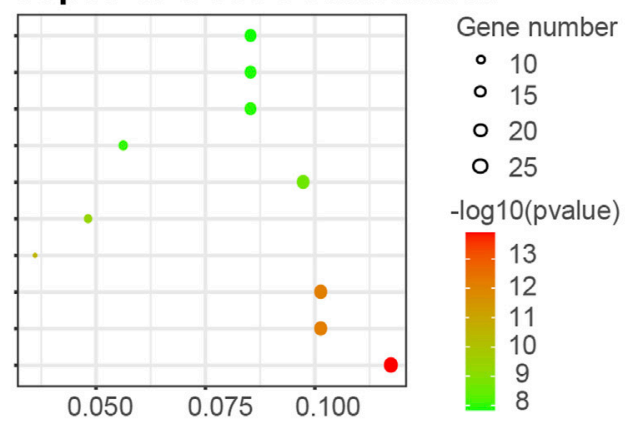

\section{Top10 of GO:MF enrichment}
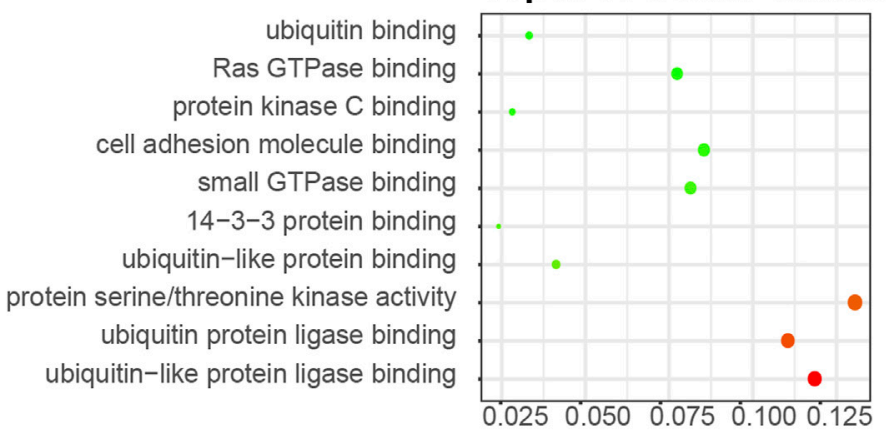

Gene number

- 10

- 15

○ 20

○ 25

○ 30

$-\log 10$ (pvalue)

15.0

12.5

10.0

7.5

5.0

\section{Top10 of kegg enrichment}

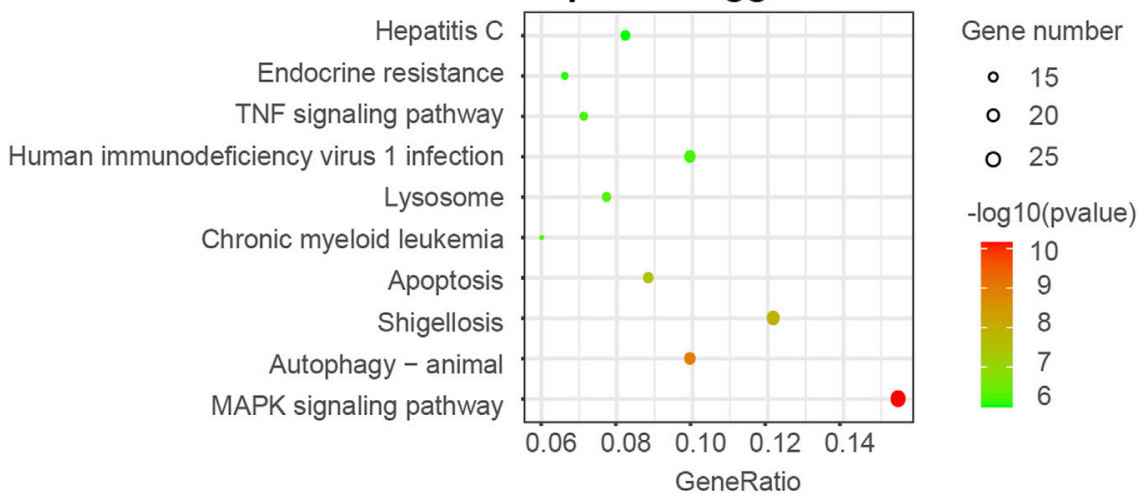

FIGURE 2 | Gene ontology (GO) and KEGG pathway enrichment analyses of ATGs in Brown (A-D) modules.

and analyzing the area under the receiver operating characteristic curve (AUC) following previous strategies (Yamamoto et al., 2013; Roy et al., 2021). As shown in Figure 6A, the area under the curve
(AUC) values of all 12 hub ATGs were more than 60\% in GSE7305. Among these, the AUC values of EZH2, SPATA18, RND3, and EPHA5 exceeded 90\%, which means that the hub genes had high 


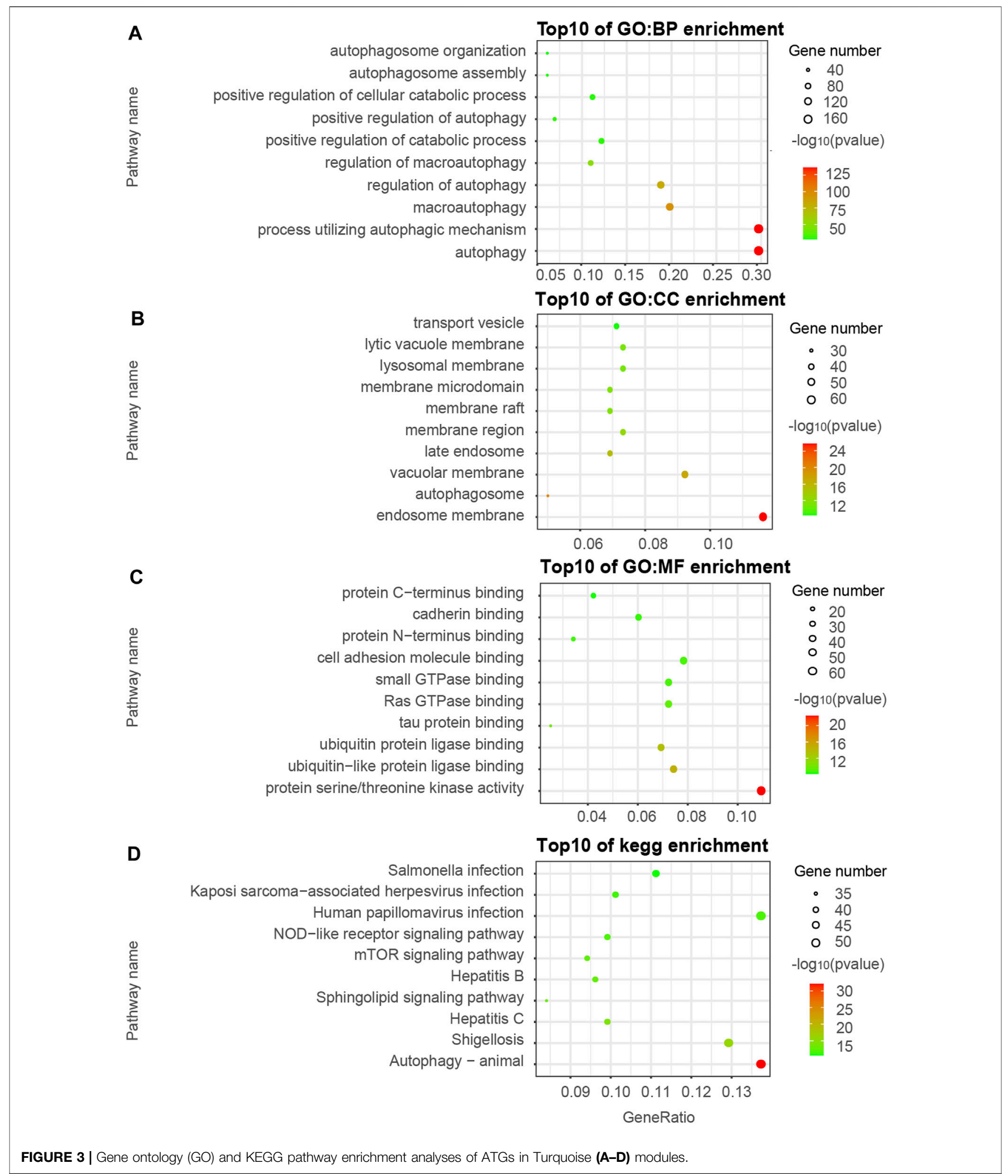

sensitivity and specificity. Cancer antigen 125 (CA125) is generally present in normal ovarian epithelia, endometrium and decidua (Liu et al., 2021), and its normal level is $<35 \mathrm{U} / \mathrm{ml}$. CA125 is often elevated in benign conditions such as endometriosis and ovarian cysts (Whitwell et al., 2020). It has been a traditional marker for ovarian tumor screening. Spearman correlation analysis depicted 
A
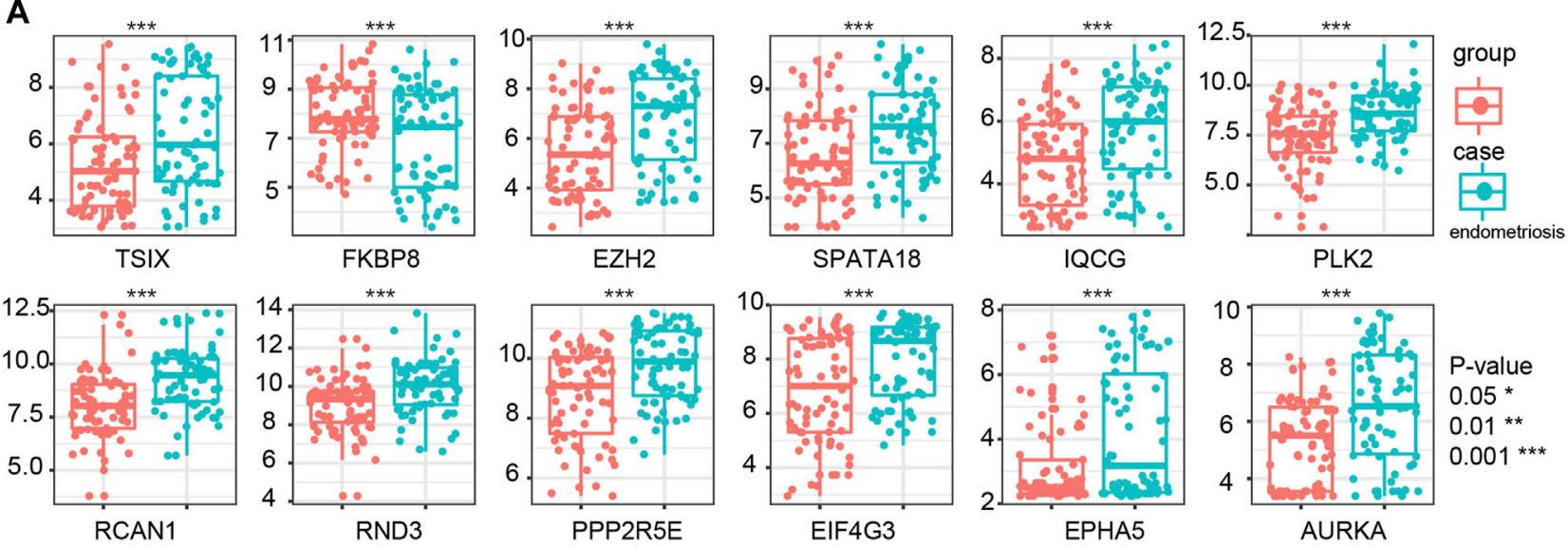

EIF4G3

C

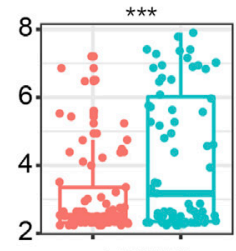

EPHA5
AURKA
B

consensus matrix $k=4$

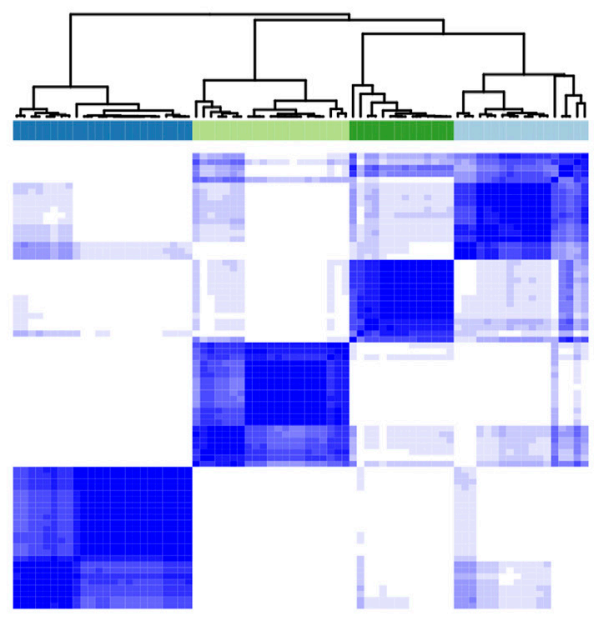

coste

cluster 1

cluste

cluster 3

(a)

cluster 4
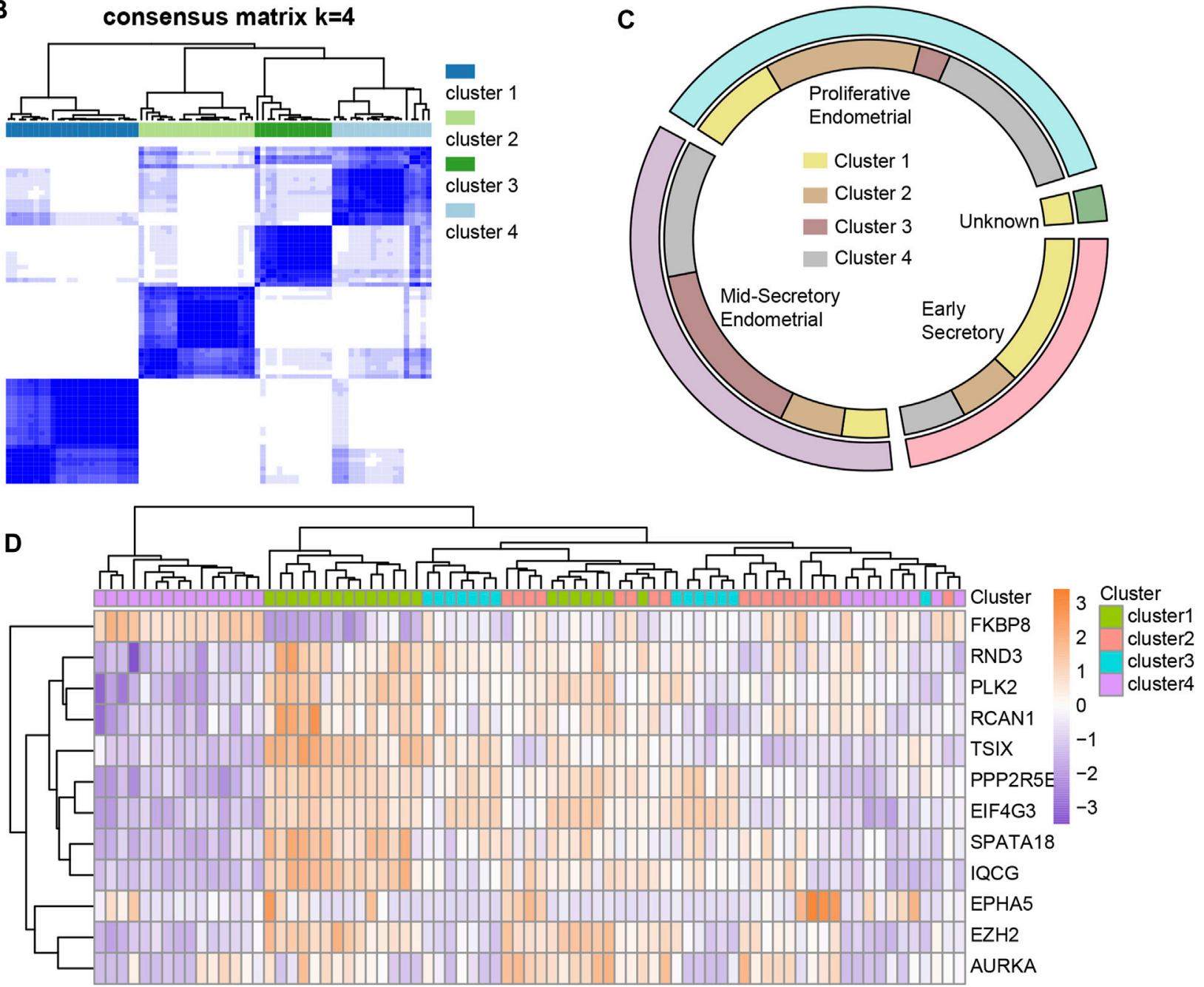

FIGURE 4 | Identification and differential expression of hub ATGs in GSE51981 (A). Four new molecular subgroups were performed by consensus clustering analysis in endometriosis (B). The distribution of the four new molecular subgroups in different periods of endometrium (C). The expression of the 12 hub ATGs in four new molecular subgroups (D) 


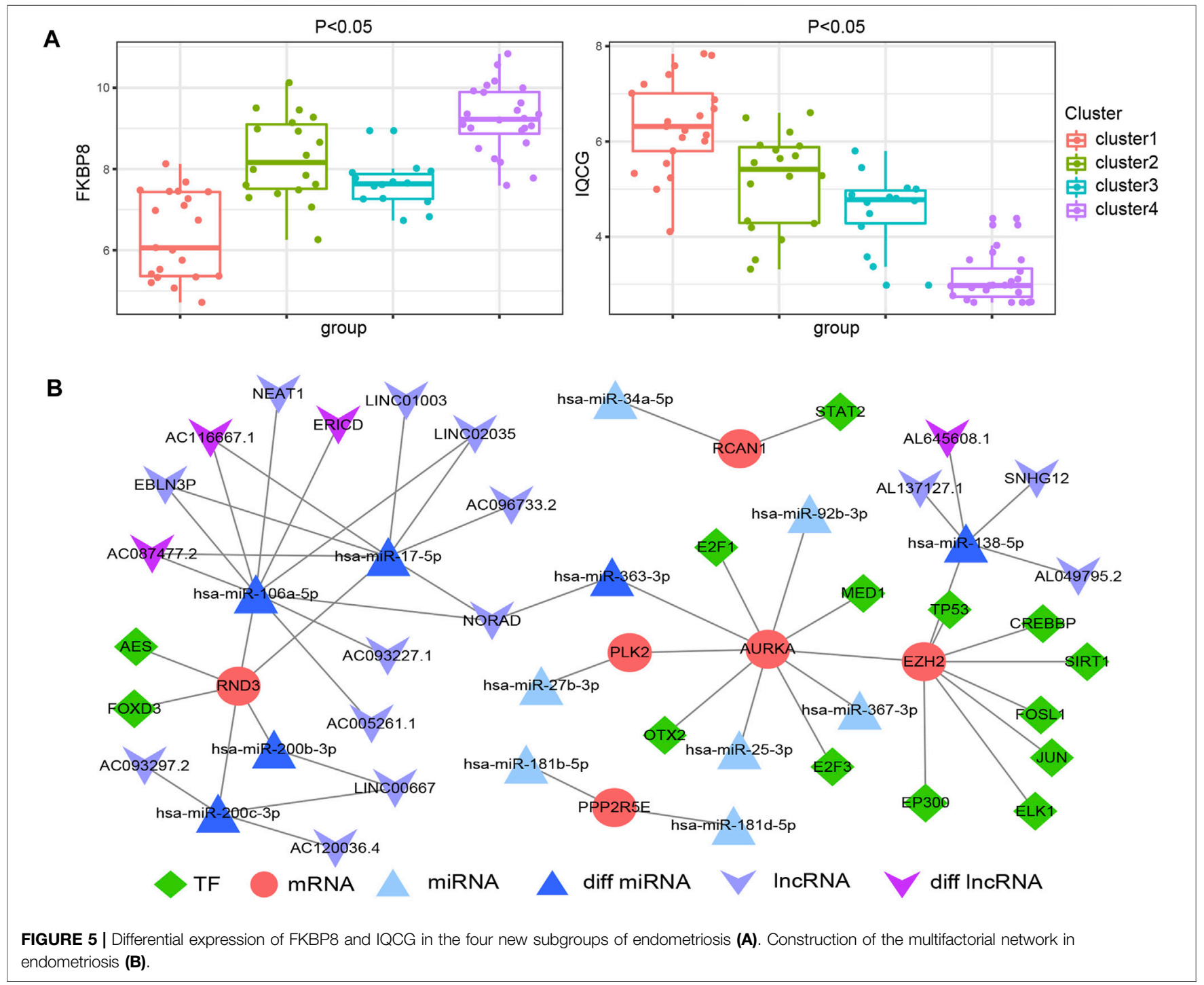

that the relative expression levels of EZH2 and RND3 were positively correlated with CA125 of the corresponding endometriosis samples, $(\mathrm{r}=0.7817, p<0.0001)$ and $(\mathrm{r}=0.7793, p<0.0001)$, respectively, (Figure 6B). These results indicated that EZH2 and RND3 could serve as novel biomarkers for endometriosis.

\section{RT-qPCR and Western Blotting Results of the Hub ATGs}

Measuring the expression of 12 hub ATGs using RT-qPCR showed that the expression levels of EZH2, IQCG, RCAN1, RND3, PPP2R5E, and EPHA5 were higher in the ectopic endometrial tissues compared with the normal endometrial tissues $(p<0.05)$, which was consistent with the expression pattern of these six genes in multiple databases (Figure 7E). Among them, the differences in the expression levels of EZH2, IQCG, and RND3 between the endometriosis group and control group were the most statistically significant $(p<0.001)$. Then, primary NESCs and EESCs were successfully isolated from the fresh normal endometrial tissues and ectopic endometrial tissues (Figures 7A,B). The purity of NESCs and EESCs was identified using cellular immunofluorescence separately for the epithelial marker Cytokeratin 7 (Abcam) and stromal marker Vimentin (Abcam) (Figures 7C,D). We only selected Vimentin cells that were positive for more than 95\% from P3-P5 for further research. The RT-qPCR results showed that the levels of EZH2, IQCG, and RND3 in EESCs were significantly higher than those in NESCs (Figure 7F). This further verified the above conclusion. In view of above results, protein expression of EZH2, RND3, and IQCG were subsequently measured in endometriosis cells and tissues by Western blotting (Figures 8A-D), which demonstrated similar results to those of the mRNA level.

\section{DISCUSSION}

In the study, the GO and KEGG enrichment analyses indicated that the ATGs were predominately associated with autophagy- 

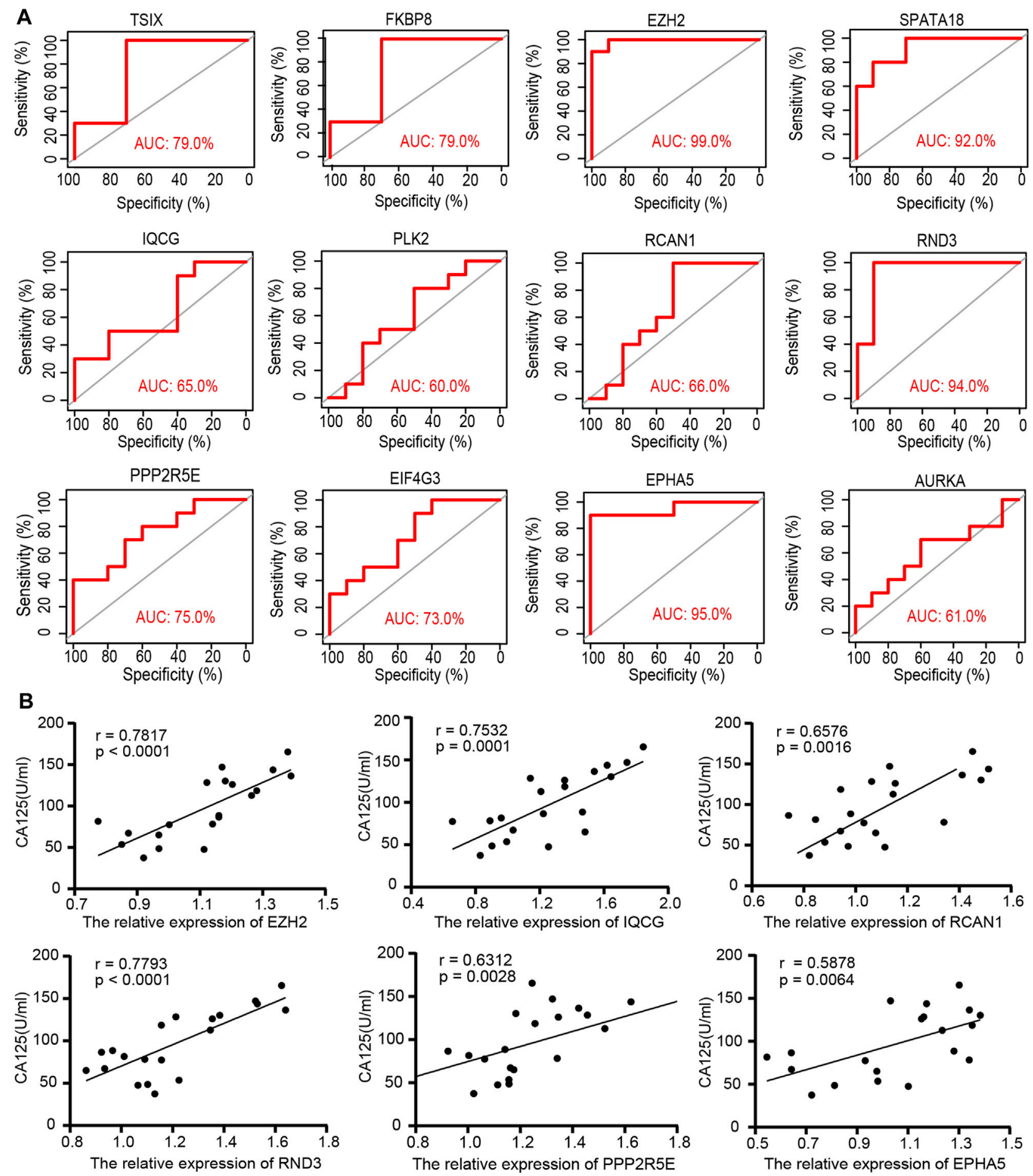

FIGURE 6 | The specificity and sensitivity of the 12 hub ATGs for the diagnosis of endometriosis by the receiver operating characteristic (ROC) analysis (A). The relative expression of the 6 hub ATGs in tissues were positively correlated with Ca125 by Spearman correlation analysis (B).

related functions and pathways, such as the processes utilizing autophagic mechanism, autophagosome, apoptosis, cell cycle, and MAPK signaling pathway. The two functions of autophagy and apoptosis are interconnected with each other
(Guo et al., 2016). In general, the most common conclusion is that basal macroautophagy was detectable at all cell cycle stages. The cell cycle regulators might directly regulate macroautophagy to accelerate the mobilization and activity of DNA damage/repair 
A

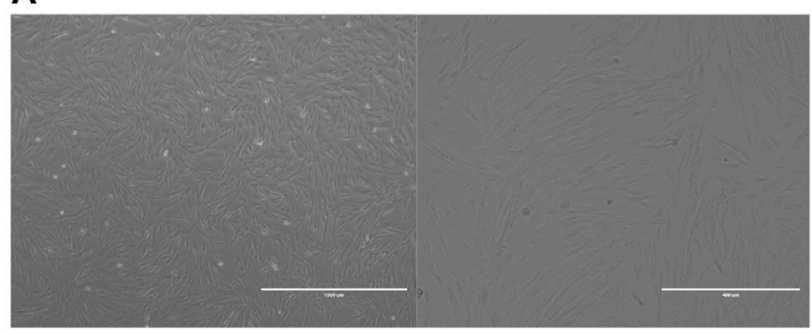

C VIM

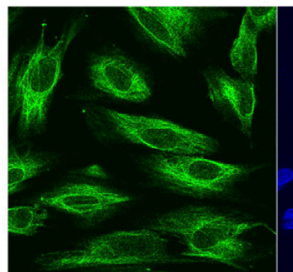

D VIM

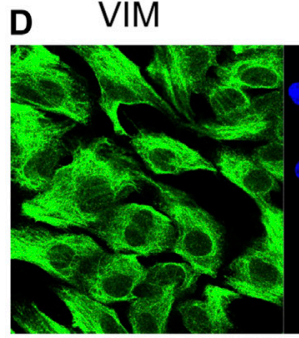

E
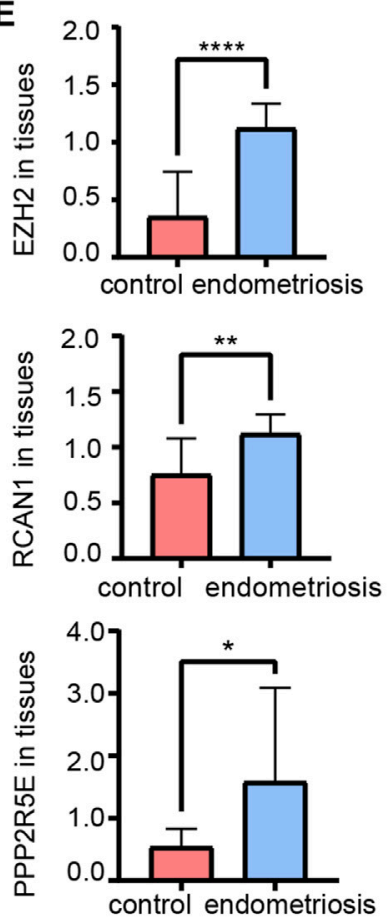

DAPI

Merge

\begin{abstract}
DAPI
Merge
\end{abstract}
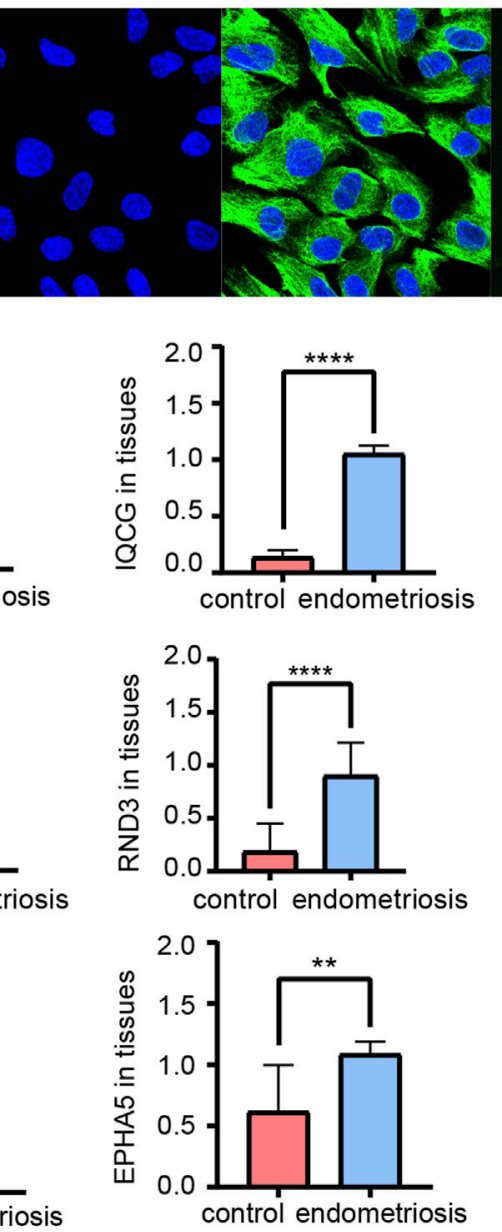

B

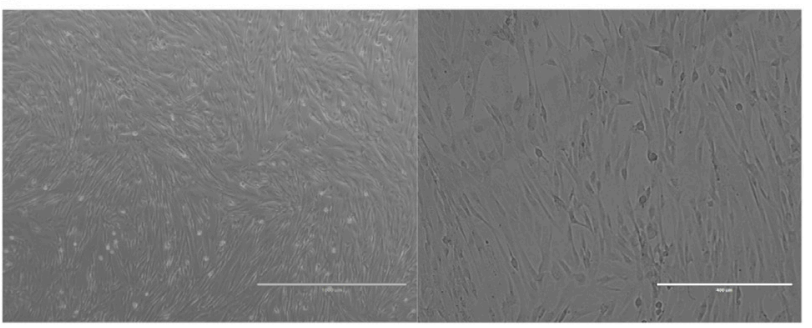

DAPI

Merge

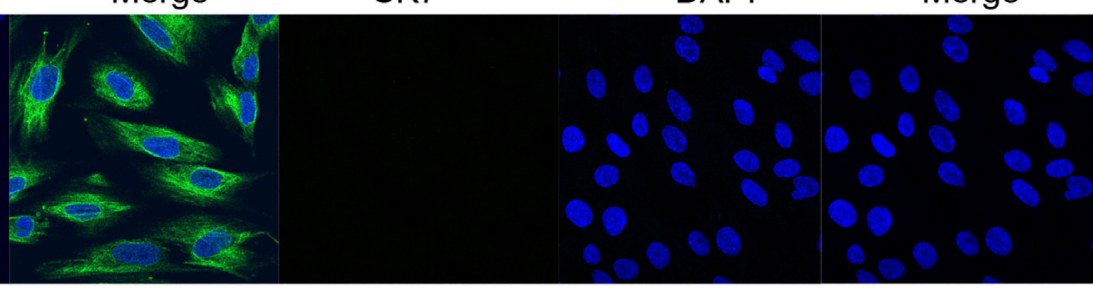

CK7

DAPI

Merge
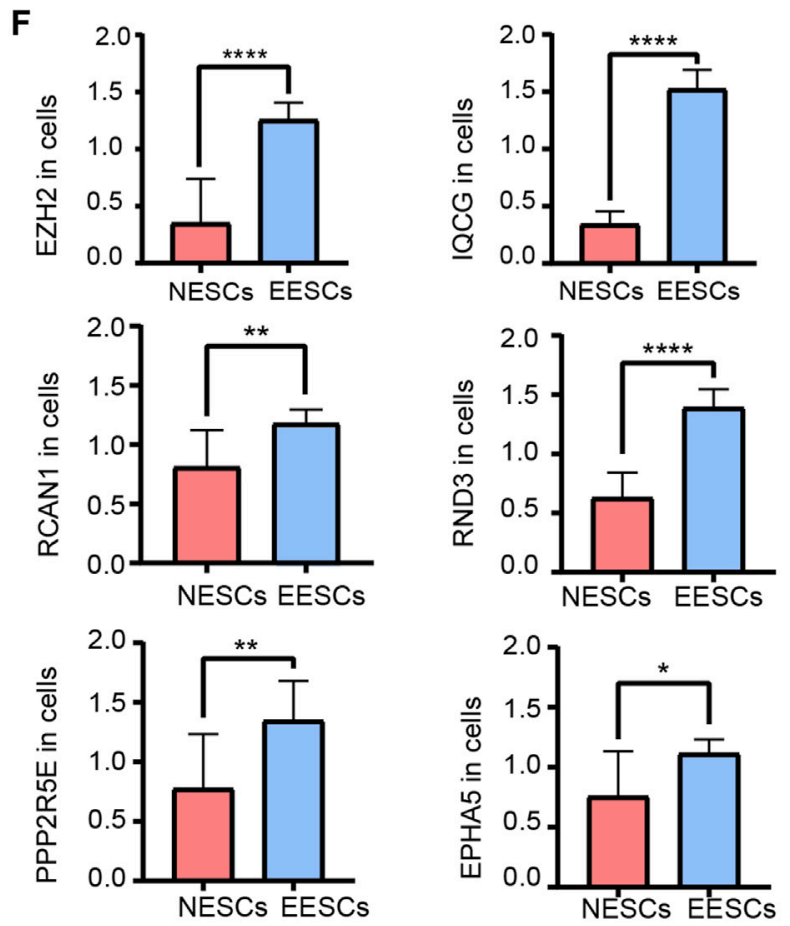

FIGURE 7 | Morphology of primary NESCs aggregates (A). Morphology of primary EESCs aggregates (B). Identification of primary NESCs (C). Identification of primary EESCs (D). The differences in the expression levels of EZH2, IQCG, RCAN1, RND3, PPP2R5E, and EPHA5 by RT-qPCR between the endometriosis tissues and the control tissues (E), those between NESCs and EESCs (F). 


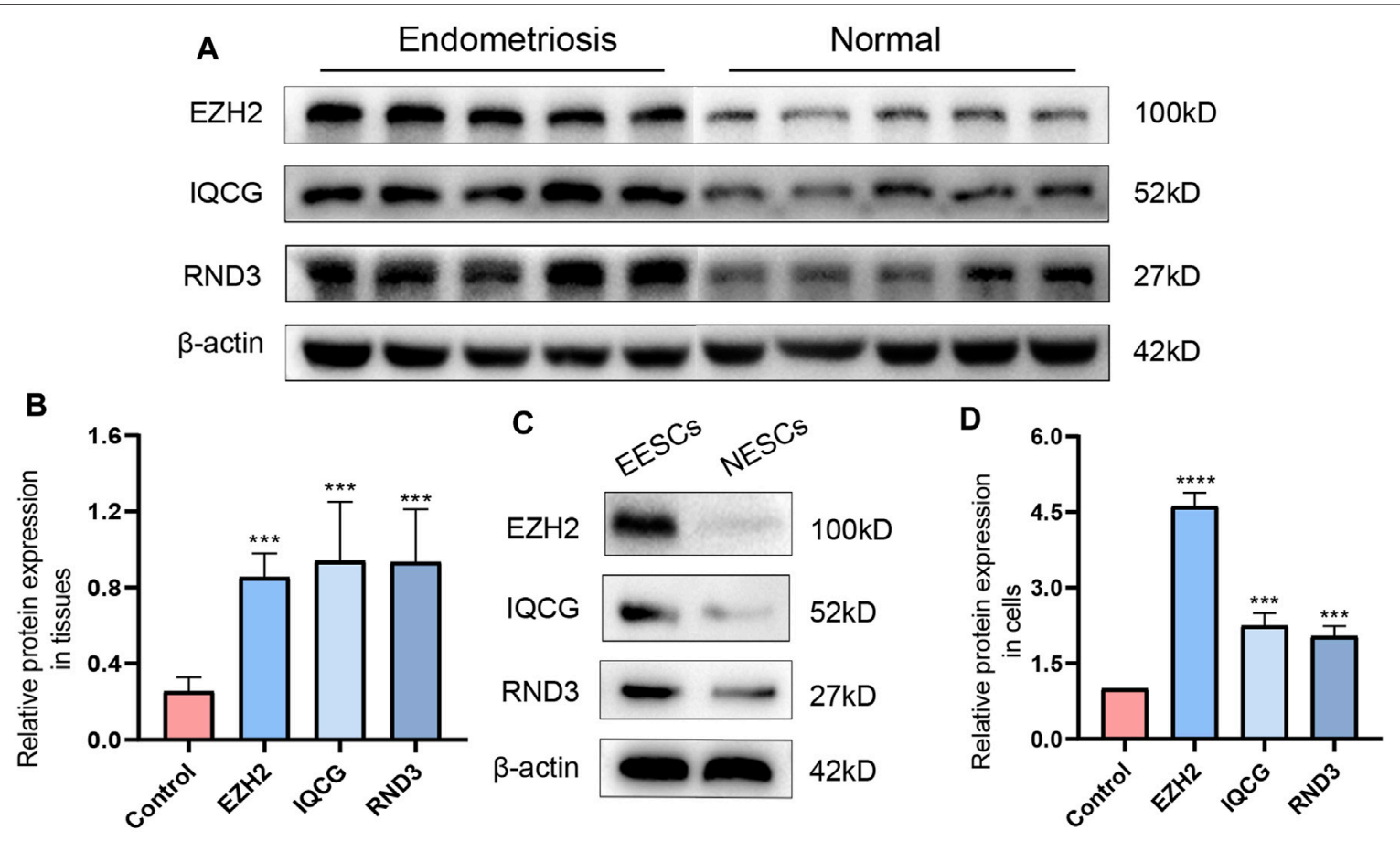

FIGURE 8 | The differences in the expression levels and quantitative analysis of EZH2, IQCG, and RND3 by Western blotting between the endometriosis tissues and the control tissues (A,B), those between NESCs and EESCs (C,D).

complex, especially in response to DNA damage (Zheng et al., 2019). In addition, it has been shown that under anoxic conditions, inhibiting autophagy by beclin1 siRNA promoted apoptosis in human endometrial stromal cells. MTOR inhibition was involved in autophagy induction to promote endometriotic cell apoptosis (Choi et al., 2014; Liu et al., 2019). In our study, GO and KEGG analysis showed that ATGs might potentially play a remarkable role in regulating autophagy and apoptosis in endometriosis. Integrin-mediated intracellular ERK/MAPK acting downstream and MAPK activation were correlated with endometriosis, which were both shown to be involved in autophagy and apoptosis, gene expression, cell division, movement, and survival (Uimari et al., 2017). Besides, a previous study revealed that iron modulated cell autophagy in a Ras and MAPK-dependent manner in ovarian cancer cells (Bauckman et al., 2013). Our findings further confirmed the involvement of ATGs in the regulation of the MAPK signaling pathways in endometriosis. This shows the importance and essentiality of conducting research on ATGs in endometrialrelated diseases. These findings have laid the foundation for the screening of new candidate biomarkers and the early diagnosis and treatment of endometriosis.

Molecular subtypes have been defined in autoimmune diseases and various cancers types. Therefore, determining the molecular subtypes of endometriosis is crucial for personalized treatment. The relationships between molecular subtypes and clinical characteristics need to be further evaluated. We performed clustering analysis of the samples in GSE51918 using ConsensusClusterPlus and further classified the endometriosis cases into 4 new molecular subgroups. This revealed that ATGs from different subgroups exhibited different clinical characteristics. Starting from cluster 1, the ATGs (EIF4G3, SPATA18, IQCG) were dominant in the early secretory phase of endometrial tissues. In cluster 3, the ATGs (mainly PPP2R5E and EIF4G3) were characterized by being dominant in the middle-secretory phase of endometrial tissues. As for cluster 2 and cluster 4, they were mainly enriched in the endometrial proliferative phase. EPHA5 is highly expressed in cluster 2 but barely expressed in the remaining clusters, which indicates a tissue-specific expression. FKBP8 was only expressed in cluster 4, while EPHA5 and FKBP8 could be substantially distinguished between cluster 2 and cluster 4 . Consequently, it seems that endometriosis tissues with different clinical characteristics could be divided into 4 new molecule subgroups. To our delight, we observed a noticeable variation trend in the expression levels of FKBP8 and IQCG in all 4 subgroups. The expression level of FKBP8 showed a dramatic upward trend from cluster 1 to cluster 4. On the contrary, IQCG manifested a pronounced downward trend from cluster 1 to cluster 4 , and distinct alterations were noticed among the 4 clusters. Hence, these sample type exclusive genes might be responsible for the early treatment of endometriosis. Next, the results of RT-qPCR and Western blotting showed that IQCG had a more pronounced difference $(p<0.001)$ between the endometriosis and control groups. Combined with the above analysis, we speculate that IQCG could be utilized to distinguish 4 new molecular subgroups.

In order to discover new valid biomarkers, we further inspected 6 hub ATGs by RT-qPCR and Western blotting in 
clinical samples and primary ESCs, 4 of which were statistically significant $(p<0.05)$. The genes with the most obvious discrepancy were EZH2 $(p<0.001)$ and RND3 $(p<0.001)$. RND3 belongs to the RND subfamily of the RHO family and has been proven to be an anti-proliferation protein. Previous studies showed that the RND3 was linked with the processes of proliferation, apoptosis, autophagy, cell cycle arrest, migration, and invasion (Paysan et al., 2016). Upregulation of RND3 suppressed the cell proliferation of gastric cancer cells and arrested them in the G0/G1 phase of the cell cycle. Additionally, in gastric cancer, chaperone-mediated autophagy contributed to cell proliferation by targeting RND3 (Zhou et al., 2016). Besides, the genetic deletion of RND3 suppressed cell apoptosis via NF-kB signaling in the brain (Dong et al., 2021). As for $\mathrm{EZH} 2$, a subunit of the Polycomb repressive complex 2 (PRC2) catalyzing trimethylation of histone $\mathrm{H} 3$ lysine 27 (H3K27), it has been shown to induce epithelial-mesenchymal transition (EMT) in several cancer types. The expression of EZH2 was obviously higher in eutopic and ectopic endometrium in the endometriosis group compared with the control group (Zhang et al., 2017). EZH2 silencing promoted autophagy in endometrial cancer cells. Meanwhile, by activating the mTOR signaling, its overexpression reduced the autophagy and survival of trophoblasts in the trophoblastic disease (Wang et al., 2018; Chu et al., 2020). Furthermore, EZH2 has been identified to be a promising tumor biomarker (Cai et al., 2011), and it represents a prognostic biomarker associated with immunosuppression in esophageal squamous carcinoma and hepatocellular carcinoma (Liu et al., 2016; Guo et al., 2020). Next, we calculated the diagnostic value of the 12 hub ATGs for endometriosis using the ROC analysis, and the results showed the high sensitivity and specificity of EZH2 (AUC: 99.0\%) and RND3 (AUC: 94.0\%) in diagnosing endometriosis. The Spearman correlation analysis results revealed EZH2, IQCG, RCAN1, RND3, PPP2R5E, and EPHA5 were positively associated with Ca125, it is worth mentioning, EZH2 and RND3 especially striking ( $\mathrm{r}>0.75, p<0.0001)$. These findings suggested that EZH2 and RND3 could be novel potential biomarkers for the diagnosis of endometriosis and further confirmed the role of hub ATGs in endometriosis.

In this work, a multifunctional co-expression network was constructed among lncRNA, miRNA, mRNA, and TF to investigate the mechanism of endometriosis. Some of the 12 hub ATGs (RND3, PLK2, AURKA, RCAN1, ppp2R5E, and EZH2) were selected to construct the lncRNA-miRNA-mRNA ceRNA network and lncRNA-TF-mRNA network. A total of 7 differentially expressed miRNAs (hsa-miR-34a-5p, hsa-miR-92b3p, hsa-miR-27b-3p, hsa-miR-181b-5p, hsa-miR-25-3p, hsamiR-367-3p, and hsa-miR-181d-5p) and 4 differentially expressed lncRNAs (AC087477.2, AC116667.1, ERICD, and AL645608.1) were overlapped with GSE105765. The genes from the multifunctional network were tightly linked and cross-regulated each other. For example, in the network, MiR138-5p/EZH2/SIRT1 were involved in the regulation of autophagy. In a cisplatin resistance of ovarian cancer cells study, the upregulation of miR-138-5p enhanced the chemosensitivity of DDP-resistant cells and apoptosis by decreasing the expression of EZH2 (Zhang et al., 2020). Another study demonstrated a binding site of EZH2/SIRT1, and showed EZH2 and SIRT1 also to participate in the regulation of autophagy. Chidamide treatment markedly downregulated histone deacetylase SIRT1, and simultaneously resulted in a dose-dependent upregulation of acetyltransferase hMOF and histone methyltransferase EZH2, which contributed to the autophagy-suppressive role of chidamide (Xu et al., 2020). At present, there are many related studies on the role of EZH2 and SIRT1 in endometriosis. EZH2 expression was significantly elevated in ectopic and eutopic endometrium from women with endometriosis compared with control endometrium (ColónCaraballo et al., 2015; Brunty et al., 2021); nuclear SIRT1 expression was increased in those with endometriosis and endometriosis-associated ovarian cancer, not in case with ovarian cancer only (Teasley et al., 2020). However, the detailed mechanism of the multifunctional network should be clarified in the future. We believe that our results will contribute to future research endeavors in this direction.

\section{CONCLUSION}

This work has shown EZH2 and RND3 to have a high value in diagnosing endometriosis and being effective diagnostic biomarkers. Multifactorial networks related to ATGs have been successfully constructed. The four new molecular subgroups of endometriosis presented in this study based on hub ATGs would assist to adapt the intervention in the future. IQCG could be used to independently differentiate among the subgroups. The above-mentioned results would be beneficial to explore the pathogenesis of endometriosis and provide the foundation to detect novel biomarkers and therapeutic targets for endometriosis. In our future work, we plan to perform an in-depth exploration of the action mechanism of ATGs and increase the sample size.

\section{DATA AVAILABILITY STATEMENT}

The original contributions presented in the study are included in the article/Supplementary Material, further inquiries can be directed to the corresponding author.

\section{ETHICS STATEMENT}

The studies involving human participants were reviewed and approved by the Ethics Commission of Harbin Medical University (202105). The patients/participants provided their written informed consent to participate in this study.

\section{AUTHOR CONTRIBUTIONS}

GZ designed and directed all research. JW, SC, HW, YH, and XL performed the data processing and experimental analyses. LS, XZ 
drafted the article. All authors reviewed and approved the final version of the article.

\section{FUNDING}

This study was supported by the National Natural Science Foundation of China (81971359); the research and development of applied technology of Harbin (2017AB9BS039); and Heilongjiang Postdoctoral Program Foundation (LBH-Z19085).

\section{ACKNOWLEDGMENTS}

Most of the experiments were performed in the State Key Laboratory of Veterinary Biotechnology, Harbin Veterinary

\section{REFERENCES}

Bauckman, K. A., Haller, E., Flores, I., and Nanjundan, M. (2013). Iron Modulates Cell Survival in a Ras- and MAPK-dependent Manner in Ovarian Cells. Cell Death Dis 4 (4), e592. doi:10.1038/cddis.2013.87

Brunty, S., Ray Wright, K., Mitchell, B., and Santanam, N. (2021). Peritoneal Modulators of EZH2-miR-155 Cross-Talk in Endometriosis. Ijms 22 (7), 3492. doi:10.3390/ijms22073492

Bulun, S. E., Wan, Y., and Matei, D. (2019). Epithelial Mutations in Endometriosis: Link to Ovarian Cancer. Endocrinology 160 (3), 626-638. doi:10.1210/en.201800794

Cai, M.-Y., Tong, Z.-T., Zheng, F., Liao, Y.-J., Wang, Y., Rao, H.-L., et al. (2011). EZH2 Protein: a Promising Immunomarker for the Detection of Hepatocellular Carcinomas in Liver Needle Biopsies. Gut 60 (7), 967-976. doi:10.1136/ gut.2010.231993

Chin, C.-H., Chen, S.-H., Wu, H.-H., Ho, C.-W., Ko, M.-T., and Lin, C.-Y. (2014). cytoHubba: Identifying Hub Objects and Sub-networks from Complex Interactome. BMC Syst. Biol. 8, S11. doi:10.1186/1752-0509-8-s4-s11

Choi, J., Jo, M., Lee, E., Kim, H. J., and Choi, D. (2014). Differential Induction of Autophagy by mTOR Is Associated with Abnormal Apoptosis in Ovarian Endometriotic Cysts. Mol. Hum. Reprod. 20 (4), 309-317. doi:10.1093/molehr/ gat091

Chu, Y., Chen, W., Peng, W., Liu, Y., Xu, L., Zuo, J., et al. (2020). Amnion-Derived Mesenchymal Stem Cell Exosomes-Mediated Autophagy Promotes the Survival of Trophoblasts under Hypoxia through mTOR Pathway by the Downregulation of EZH2. Front. Cel Dev. Biol. 8, 545852. doi:10.3389/ fcell.2020.545852

Colón-Caraballo, M., Monteiro, J. B., and Flores, I. (2015). H3K27me3 Is an Epigenetic Mark of Relevance in Endometriosis. Reprod. Sci. 22 (9), 1134-1142. doi:10.1177/1933719115578924

Dong, H., Sun, Q., Zhang, Y., Li, Y., Yuan, F. e., Mao, S., et al. (2021). Genetic Deletion of Rnd3 Suppresses Apoptosis through NF- $\kappa B$ S-ignaling in the B-rain. Oncol. Rep. 45 (2), 595-605. doi:10.3892/or.2020.7884

Gargett, C. E., Schwab, K. E., Zillwood, R. M., Nguyen, H. P. T., and Wu, D. (2009). Isolation and Culture of Epithelial Progenitors and Mesenchymal Stem Cells from Human Endometrium1. Biol. Reprod. 80 (6), 1136-1145. doi:10.1095/ biolreprod.108.075226

Guo, B., Tan, X., and Cen, H. (2020). EZH2 Is a Negative Prognostic Biomarker Associated with Immunosuppression in Hepatocellular Carcinoma. PLoS ONE 15 (11), e0242191. doi:10.1371/journal.pone.0242191

Guo, R., Lin, B., Pan, J. F., Liong, E. C., Xu, A. M., Youdim, M., et al. (2016). Inhibition of Caspase-9 Aggravates Acute Liver Injury through Suppression of Cytoprotective Autophagy. Sci. Rep. 6, 32447. doi:10.1038/srep32447

He, R., Liu, X., Zhang, J., Wang, Z., Wang, W., Fu, L., et al. (2020). NLRC5 Inhibits Inflammation of Secretory Phase Ectopic Endometrial Stromal Cells by UpRegulating Autophagy in Ovarian Endometriosis. Front. Pharmacol. 11, 1281. doi:10.3389/fphar.2020.01281
Research Institute, Chinese Academy of Agricultural Sciences. The authors thank Dr. Xiaojun Wang for his contributions regarding experimental guidance. The authors express their gratitude to EditSprings (https:// www.editsprings.com/) for the expert linguistic services provided.

\section{SUPPLEMENTARY MATERIAL}

The Supplementary Material for this article can be found online at: https://www.frontiersin.org/articles/10.3389/fmolb.2021.743012/ full\#supplementary-material

Huang, H., Zhang, Q., Ye, C., Lv, J.-M., Liu, X., Chen, L., et al. (2017). Identification of Prognostic Markers of High Grade Prostate Cancer through an Integrated Bioinformatics Approach. J. Cancer Res. Clin. Oncol. 143 (12), 2571-2579. doi:10.1007/s00432-017-2497-0

Langfelder, P., and Horvath, S. (2012). Fast R Functions for Robust Correlations and Hierarchical Clustering. J. Stat. Softw. 46 (11). doi:10.18637/jss.v046.i11

Langfelder, P., and Horvath, S. (2008). WGCNA: an R Package for Weighted Correlation Network Analysis. BMC Bioinformatics 9, 559. doi:10.1186/14712105-9-559

Liu, F., Gu, L., Cao, Y., Fan, X., Zhang, F., and Sang, M. (2016). Aberrant Overexpression of EZH2 and H3K27me3 Serves as Poor Prognostic Biomarker for Esophageal Squamous Cell Carcinoma Patients. Biomarkers 21 (1), 80-90. doi:10.3109/1354750x.2015.1118537

Liu, H., Zhang, Z., Xiong, W., Zhang, L., Du, Y., Liu, Y., et al. (2019). Long Noncoding RNA MALAT1 Mediates Hypoxia-Induced Pro-survival Autophagy of Endometrial Stromal Cells in Endometriosis. J. Cel Mol Med 23 (1), 439-452. doi:10.1111/jcmm.13947

Liu, J., Li, L., Luo, N., Liu, Q., Liu, L., Chen, D., et al. (2021). Inflammatory Signals Induce MUC16 Expression in Ovarian Cancer Cells via NF- $\kappa$ B A-ctivation. Exp. Ther. Med. 21 (2), 163. doi:10.3892/etm.2020.9594

Liu, W., Li, L., Ye, H., and Tu, W. (2017). Weighted Gene Co-expression Network Analysis in Biomedicine Research. Sheng Wu Gong Cheng Xue Bao 33 (11), 1791-1801. doi:10.13345/j.cjb.170006

Love, M. I., Huber, W., and Anders, S. (2014). Moderated Estimation of Fold Change and Dispersion for RNA-Seq Data with DESeq2. Genome Biol. 15 (12), 550. doi:10.1186/s13059-014-0550-8

Luo, X., Cheng, W., Wang, S., Chen, Z., and Tan, J. (2018). Autophagy Suppresses Invasiveness of Endometrial Cells through Reduction of Fascin-1. Biomed. Res. Int. 2018, 1-9. doi:10.1155/2018/8615435

Ma, L., Li, Z., Li, W., Ai, J., and Chen, X. (2019). MicroRNA-142-3p Suppresses Endometriosis by Regulating KLF9-Mediated Autophagy In Vitro and In Vivo. RNA Biol. 16 (12), 1733-1748. doi:10.1080/15476286.2019.1657352

Mei, J., Zhou, W.-J., Zhu, X.-Y., Lu, H., Wu, K., Yang, H.-L., et al. (2018). Suppression of Autophagy and HCK Signaling Promotes PTGS2high FCGR3- NK Cell Differentiation Triggered by Ectopic Endometrial Stromal Cells. Autophagy 14 (8), 1376-1397. doi:10.1080/15548627.2018.1476809

Pascut, D., Pratama, M. Y., Gilardi, F., Giuffrè, M., Crocè, L. S., and Tiribelli, C. (2020). Weighted miRNA Co-expression Networks Analysis Identifies Circulating miRNA Predicting Overall Survival in Hepatocellular Carcinoma Patients. Sci. Rep. 10 (1), 18967. doi:10.1038/s41598-020-75945-2

Paysan, L., Piquet, L., Saltel, F., and Moreau, V. (2016). Rnd3 in Cancer: A Review of the Evidence for Tumor Promoter or Suppressor. Mol. Cancer Res. 14 (11), 1033-1044. doi:10.1158/1541-7786.Mcr-16-0164

Ritchie, M. E., Phipson, B., Wu, D., Hu, Y., Law, C. W., Shi, W., et al. (2015). Limma powers Differential Expression Analyses for RNA-Sequencing and Microarray Studies. Nucleic Acids Res. 43 (7), e47. doi:10.1093/nar/gkv007

Rižner, T. L. (2009). Estrogen Metabolism and Action in Endometriosis. Mol. Cell Endocrinol. 307 (1-2), 8-18. doi:10.1016/j.mce.2009.03.022 
Roy, P.-M., Friou, E., Germeau, B., Douillet, D., Kline, J. A., Righini, M., et al. (2021). Derivation and Validation of a 4-Level Clinical Pretest Probability Score for Suspected Pulmonary Embolism to Safely Decrease Imaging Testing. JAMA Cardiol. 6 (6), 669-677. doi:10.1001/jamacardio.2021.0064

Teasley, H. E., Beesley, A., Kim, T. H., Risinger, J., Young, S. L., Jeong, J.-W., et al. (2020). Differential Expression of KRAS and SIRT1 in Ovarian Cancers with and without Endometriosis. Reprod. Sci. 27 (1), 145-151. doi:10.1007/s43032019-00017-4

Uimari, O., Rahmioglu, N., Nyholt, D. R., Vincent, K., Missmer, S. A., Becker, C., et al. (2017). Genome-wide Genetic Analyses Highlight Mitogen-Activated Protein Kinase (MAPK) Signaling in the Pathogenesis of Endometriosis. Hum. Reprod. 32 (4), 780-793. doi:10.1093/humrep/dex024

Vallvé-Juanico, J., Houshdaran, S., and Giudice, L. C. (2019). The Endometrial Immune Environment of Women with Endometriosis. Hum. Reprod. Update 25 (5), 565-592. doi:10.1093/humupd/dmz018

Wang, C., and Liu, B. (2018). miR-101-3p Induces Autophagy in Endometrial Carcinoma Cells by Targeting EZH2. Arch. Gynecol. Obstet. 297 (6), 1539-1548. doi:10.1007/s00404-018-4768-7

Whitwell, H. J., Worthington, J., Blyuss, O., Gentry-Maharaj, A., Ryan, A., Gunu, R., et al. (2020). Improved Early Detection of Ovarian Cancer Using Longitudinal Multimarker Models. Br. J. Cancer 122 (6), 847-856. doi:10.1038/s41416-019-0718-9

Wilkerson, M. D., and Hayes, D. N. (2010). ConsensusClusterPlus: a Class Discovery Tool with Confidence Assessments and Item Tracking. Bioinformatics 26 (12), 1572-1573. doi:10.1093/bioinformatics/btq170

Xu, L., Feng, J., Tang, H., Dong, Y., Shu, M., Chen, X., et al. (2020). Chidamide Epigenetically Represses Autophagy and Exerts Cooperative Antimyeloma Activity with Bortezomib. Cel Death Dis 11 (4), 297. doi:10.1038/s41419020-2414-3

Yamamoto, E., Tomita, N., Sakata, S., Tsuyama, N., Takeuchi, K., Nakajima, Y., et al. (2013). MIB-1 Labeling index as a Prognostic Factor for Patients with Follicular Lymphoma Treated with Rituximab Plus CHOP Therapy. Cancer Sci. 104 (12), 1670-1674. doi:10.1111/cas.12288

Yang, F., Chen, E., Yang, Y., Han, F., Han, S., Wu, G., et al. (2019a). The Akt/FoxO/ p27 Kip1 axis Contributes to the Anti-proliferation of Pentoxifylline in Hypertrophic Scars. J. Cel Mol Med 23 (9), 6164-6172. doi:10.1111/jcmm.14498

Yang, H., Mei, J., Chang, K., Zhou, W., Huang, L., and Li, M. (2017). Autophagy in Endometriosis. Am. J. Translational Res. 9 (11), 4707-4725.

Yang, S., Wang, H., Li, D., and Li, M. (2019b). Role of Endometrial Autophagy in Physiological and Pathophysiological Processes. J. Cancer 10 (15), 3459-3471. doi:10.7150/jca.31742
Yepes, S., López, R., Andrade, R. E., Rodriguez-Urrego, P. A., López-Kleine, L., and Torres, M. M. (2016). Co-expressed miRNAs in Gastric Adenocarcinoma. Genomics 108 (2), 93-101. doi:10.1016/j.ygeno.2016.07.002

Zhang, Q., Dong, P., Liu, X., Sakuragi, N., and Guo, S.-W. (2017). Enhancer of Zeste Homolog 2 (EZH2) Induces Epithelial-Mesenchymal Transition in Endometriosis. Sci. Rep. 7 (1), 6804. doi:10.1038/s41598-017-06920-7

Zhang, Y., Ai, H., Fan, X., Chen, S., Wang, Y., and Liu, L. (2020). Knockdown of Long Non-coding RNA Hotair Reverses Cisplatin Resistance of Ovarian Cancer Cells through Inhibiting miR-138-5p-Regulated EZH2 and SIRT1. Biol. Res. 53 (1), 18. doi:10.1186/s40659-020-00286-3

Zhao, Q., Ye, M., Yang, W., Wang, M., Li, M., Gu, C., et al. (2018). Effect of Mst1 on Endometriosis Apoptosis and Migration: Role of Drp1-Related Mitochondrial Fission and Parkin-Required Mitophagy. Cell Physiol Biochem 45 (3), 1172-1190. doi:10.1159/000487450

Zheng, K., He, Z., Kitazato, K., and Wang, Y. (2019). Selective Autophagy Regulates Cell Cycle in Cancer Therapy. Theranostics 9 (1), 104-125. doi:10.7150/ thno.30308

Zhou, J., Yang, J., Fan, X., Hu, S., Zhou, F., Dong, J., et al. (2016). Chaperonemediated Autophagy Regulates Proliferation by Targeting RND3 in Gastric Cancer. Autophagy 12 (3), 515-528. doi:10.1080/15548627.2015.1136770

Zhou, L., Du, Y., Kong, L., Zhang, X., and Chen, Q. (2018). Identification of Molecular Target Genes and Key Pathways in Hepatocellular Carcinoma by Bioinformatics Analysis. OncoTargets Ther. 11, 1861-1869. doi:10.2147/ ott.S156737

Conflict of Interest: The authors declare that the research was conducted in the absence of any commercial or financial relationships that could be construed as a potential conflict of interest.

Publisher's Note: All claims expressed in this article are solely those of the authors and do not necessarily represent those of their affiliated organizations, or those of the publisher, the editors and the reviewers. Any product that may be evaluated in this article, or claim that may be made by its manufacturer, is not guaranteed or endorsed by the publisher.

Copyright (C) 2021 Wang, Cong, Wu, He, Liu, Sun, Zhao and Zhang. This is an openaccess article distributed under the terms of the Creative Commons Attribution License (CC BY). The use, distribution or reproduction in other forums is permitted, provided the original author(s) and the copyright owner(s) are credited and that the original publication in this journal is cited, in accordance with accepted academic practice. No use, distribution or reproduction is permitted which does not comply with these terms. 